UA/NPPS-9-98

\title{
Worldline Approach to Forward and Fixed Angle fermion-fermion Scattering in Yang-Mills Theories at High Energies
}

\author{
A. I. Karanikas and C. N. Ktorides \\ University of Athens, Department of Physics \\ Nuclear and Particle Physics Section \\ Panepistimiopolis \\ GR-15771 Athens, Greece
}

June, 1998

\begin{abstract}
Worldline techniques are employed to study the general behaviour of the fermionfermion collision amplitude at very high energies in a non-abelian gauge field theory for the forward and fixed angle scattering cases. A central objective of this work is to demonstrate the simplicity by which the worldline methodology isolates that sector of the full theory which carries the soft physics, relevant to each process. Anomalous dimensions pertaining to a given soft sector are identified and subsequently used to facilitate the renormalization group running of the respective four point functions. Gluon reggeization is achieved for forward, while Sudakov behaviour is established for fixed angle scattering.
\end{abstract}

PACS numbers: 11.80.Fv, 12.38.Cy. 11.10.Jj 


\section{Introduction}

The theoretical confrontation of collision experiments at high energies calls for methodologies that have an essential dependence on the kinematics of the process. Fixing our ideas on investigations addressing themselves to hadronic structure -even though references to QED, whenever relevant, will also be made in this work- one of the key issues involved concerns the interplay between the (invariant) center of mass energy $\sqrt{s}$, on the one hand, and the (invariant) momentum transfer $\sqrt{-t}$, on the other. Asymptotic regimes corresponding to the cases: (a) $|t|$ large $-\frac{t}{2 p q}, p$ proton and $q$ photon four-momentum, fixed (Bjorken limit) and (b) $s$ large $-t$ fixed (Regge limit) have come under extensive theoretical scrutiny, especially in connection with deep inelastic scattering (DIS) processes.

Given that momentum transfer defines the resolution by which the short distance structure of a hadron (nucleon) is being probed, the Bjorken limit has naturally taken historical precedence in both the experimental and the theoretical front. More recently, the Regge kinematical regime has been receiving wide attention in view of the ongoing experiments at HERA. When the emphasis on asymptotics shifts to $s$, it is the hadronic profile imprinted onto the plane transverse to the direction of the collision that forms the basis of theoretical interest. In a perturbative context, which one readily adopts by taking $-t \gg \Lambda_{Q C D}^{2}$, the emerging picture is that of a high density distribution of partons accross the surface perpendicular to the motion, each carrying a small fraction $x(\ll 1)$ of the hadron momentum. In such a context the dynamics pertaining to the process is studied in the two dimensional transverse plane where, once again, momentum transfer facilitates the probing of the hadron. The situation just described fits exactly into an eikonal mode of description wherein the no impulse approximation forces the momentum of the exchanged quanta to have (appreciably) non-vanishing components only in the transverse direction.

The most systematic quantitative considerations, referring to the qualitative account given above in connection with the $s-t$ interplay, have been made, within the framework of perturbation theory, by Cheng and $\mathrm{Wu}[1]$ for both abelian and non-abelian gauge systems with spin-1/2 matter fields. Special emphasis, in the work of these authors, was placed on the issue of unitarity which calls for separate attention to the $s$ and the $t$ channel, respectively. The so called Cheng-Wu towers, in QED, which stretch along the $t$ (vertical) 
direction present, upon cutting, a fragmented profile of the electron (positron), see Fig. 1a. In non-abelian theories, on the other hand, Cheng and Wu discover reggeization of the gluon exchange among the colliding particles. The corresponding dominant towers that unfold along the $t$-channel are formed by the exchange of gluons between reggeons, see Fig. $1 \mathrm{~b}$.

In addition to the Bjorken and Regge limits a third situation which presents interest, all of its own, is defined by the specification $s,|t| \rightarrow \infty$ at fixed ratio $s / t$. We shall henceforth refer to it as fixed angle kinematical regime, implying that the angle is fairly wide and that the collision energy is very large. With respect to DIS we expect this case to be relevant in semi-inclusive processes, when the observed particle in the final state emerges at an angle with respect to the (virtual) photon-nucleon direction.

Having said the above let us define the bounds of the present work by specifying that what we intend to pursue is the problem of the non-abelian scattering among fundamental spin-1/2 fields, cf. isolated quarks, in the Regge and fixed angle kinematical regimes. Our main objective is to show how the worldline casting of field systems, which we have been systematically pursuing in recent years [2-6], leads to efficient and straight forward methods of calculation, considerably simpler than corresponding procedures developed within the Feynmann diagrammatic description of QCD. We place particular significance on the issue of factorization between soft and hard physics entering a given process of interest which, next to confinement, is the most important aspect of QCD applications -certainly the one that lends itself to present day capabilities in coping with the theory.

It becomes obvious that, in this work, we shall neither venture into the domain of (exclusive) hadron-hadron scattering, where phenomenologically-based factorization issues [7-10] involving hadronic wave functions $[11,12]$ become essential, nor into DIS matters where structure functions and evolution equations [13,14] assume primary role. Clearly, such undertakings pose ultimate goals of pursuit and set directions for future work. Our main concern, presently, is the identification of global behaviors -as opposed to investigations of structure- underlying each of the amplitudes we are interested in, i.e. what one naturally associates with the soft physics.

The basic advantage of the worldline casting of a theory, such as QCD, is the spacetime setting that underlines the scheme as a whole [15-17,2,3]. This facilitates considerations 
which, being much closer to intuitive, geometrical pictures, as opposed to what one can associate with Feynman diagrams, lead to efficient computational procedures [16-18,3-6]. Considerations of similar nature have already been evidenced in the work of several authors in connection with the study of Wilson loops [19-23] which, after all, correspond to (Euclidean) "worldline" contours of infinitely heavy matter fields. The difference, at a foundational level, is that in our case Wilson loops/lines enter as natural ingredients of the field theoretical description of the system per se-see, e.g., [4]- and not as part of an operator-based formalism -see,e.g., [24,25]. A practical consequence of this occurence is our ability to deal with Wilson line operators of finite length. As shown in [6], manipulations with open Wilson lines (of finite extent) in worldline formalism when applied to forward QED processes are naturally associated with off mass shell eikonals. We intend to capitalize on this fact in the present paper using it to achieve infrared regularized expressions by going off mass shell.

Renormalization issues will also play a central role in our subsequent analysis. As already alluded to above, our main preoccupation is to isolate a sector of the full Yang-Mills field system wherein the active degrees of freedom are the soft ones. Even within such a subsector, the disparity between its upper momentum cutoff and the infrared one' gives rise to anomalous dimensions, having appropriate interpretations. Our first illustration of the situation in hand will be presented in connection with the forward amplitude, in the $s \rightarrow \infty$, $t$ fixed kinematical region. An immediate application of the resulting structure will be the establishement, in the LLA, of the reggeization of non-abelian gauge fields exchanged among the colliding fermions. This will be readily accomplished via a renormalization group running within the soft subtheory, which employs the abovementioned anomalous dimensions $[26]$.

A more demanding task is posed by the fixed angle scattering process. The bulk of our efforts in this case will fall upon factorization issues which become more compelling, in comparison to the previous (Regge) one, due to the presence of a hard momentum scale. The idea is to first identify the relevant anomalous dimensions in the, factorized, soft sector

\footnotetext{
${ }^{1}$ It is important to distiguish between what one terms 'soft' and what 'infrared': The first characterization pertains to observable degrees of freedom while the second refers to unobservable ones which presumably reside in the 'vacuum' state.
} 
and then proceed to derive the expression for the whole amplitude, in the LLA, exploiting its invariance with respect to the scale which separates soft from hard components. As it turns out, the end result of this analysis is the emergence of a Sudakov $[27,28]$ suppression factor which dampens the four-point process.

The particulars of our worldline casting of field systems have been extensively discussed in several papers [2-6], so the interested reader should refer to these sources, especially [3]. Suffice it to say that such a casting amounts to a reformulation $\int \mathcal{D} \bar{\psi}(x) \mathcal{D} \psi(x) e^{S\left[\bar{\psi}(x), \psi(x), A_{\mu}(x)\right]} \ldots \longrightarrow$ $\int \mathcal{D} x(\tau) \mathcal{D} p(\tau) e^{S\left[x(\tau), p(\tau), A_{\mu}(x(\tau))\right]} \ldots$, taking us from a functional to a path integral description of the system. Note that the above transcription pertains to the fermionic sector of the gauge field theory which registers via quadratic terms in the action. Accordingly, nothing is lost as one carries out the Gaussian-Grassmannian integrations over the corresponding fields. Functional integration with respect to the gauge fields remains to be carried out and it is within this context that the dynamics operating in the system reveals itself.

The organization of our paper is straight forward: In Section 2 we deal with Regge limit behaviour, while in Section 3 we study the fixed angle kinematical regime for (isolated) fermion-fermion scattering in a non-abelian gauge field system. (The extraction of two expressions entering the soft part of the fixed angle amplitude is traced in an Appendix.) For the convenience of the reader each section is divided into subsections, addressing respective issues in a self-contained manner. A final, brief, section is devoted to conclusions and outlook.

\section{Non-abelian Scattering Amplitude at Regge Asymptotics}

In this section we shall consider the scattering of two spin- $1 / 2$ particles belonging to the fundamental representation of a given non-abelian gauge, $S U(N)$, group in the limit $s \rightarrow$ $\infty,|t|\left(\gg \Lambda_{Q C D}^{2}\right)$ fixed. As already stated, we shall employ a worldline mode of description for the process in the context of which the eikonal approximation acquires a sharp, geometricallybased, interpretation. Our efforts will address the amplitude directly. Special emphasis will be placed on our ability to control IR divergencies by going off-shell whereupon one deals with open Wilson line operators?2. A renormalization group running which leads to gluon

\footnotetext{
${ }^{2}$ By contrast, the on mass-shell alternative, which calls for an explicit gluon mass as IR regulator, corresponds to the employment of Wilson loop operators.
} 
reggeization will be carried out, while some specific remarks pertinent to QED will be made in the end.

\subsection{Four-point function in worldline formalism}

Working in Euclidean space we introduce the four-point function

$$
\begin{aligned}
& \left.\frac{\delta^{4}}{\delta \bar{\eta}_{i}^{f}\left(x_{1}\right) \delta \bar{\eta}_{j}^{f}\left(x_{2}\right) \delta \eta_{i^{\prime}}^{f}\left(y_{1}\right) \delta \eta_{j^{\prime}}^{f}\left(y_{2}\right)} \quad \ln Z(\bar{\eta}, \eta)\right|_{\bar{\eta}=\eta=0} \\
& =\mathcal{M}\left(x_{1}, x_{2} ; y_{1}, y_{2}\right)_{j j^{\prime}}^{i i^{\prime}}-\mathcal{M}\left(x_{1}, x_{2} ; y_{1}, y_{2}\right)_{j i^{\prime}}^{i j^{\prime}}
\end{aligned}
$$

where $Z(\bar{\eta}, \eta)$ is the partition function for the non-abelian gauge field theory with spin-1/2 matter fields whose sources are $\bar{\eta}, \eta$, while $f$ is a flavor and $i, j \ldots$ are group representation indices. The two, connected, four-point amplitudes on the right hand side are related via a particle exchange in the final state. The second of the two terms, however, gives little contribution to the forward direction scattering process, which presently interests us, so its presence will be ignored for the rest of this section.

The expression for the amplitude in worldline formalism is

$$
\begin{aligned}
\mathcal{M}_{j j^{\prime}}^{i i^{\prime}}=\sum_{C_{x_{1}, x_{2}}^{I}} \sum_{C_{x_{2}, y_{2}}^{I I}} I\left[\dot{x}^{I}\right] I\left[\dot{x}^{I I}\right] & <\operatorname{Pexp}\left[i g \int_{0}^{T_{1}} d \tau \dot{x}^{I}(\tau) \cdot A\left(x^{I}(\tau)\right)\right]_{i i^{\prime}} \\
& \times \operatorname{Pexp}\left[i g \int_{0}^{T_{2}} d \tau \dot{x}^{I I}(\tau) \cdot A\left(x^{I I}(\tau)\right)\right]_{j j^{\prime}}>_{A}^{\text {conn }}
\end{aligned}
$$

where

$$
\begin{aligned}
\sum_{C_{x, y}^{I}} I[\dot{x}] \equiv \quad & \int_{0}^{\infty} d T \int_{\substack{x(0)=x \\
x(T)=y}} \mathcal{D} x(\tau) \int \mathcal{D} p(\tau) P \exp \left[-\int_{0}^{T} d \tau\left(i p(\tau) \cdot \gamma+m_{f}\right)\right] \\
& \times \exp \left[i \int_{0}^{T} d \tau p(\tau) \cdot \dot{x}(\tau)\right]
\end{aligned}
$$

Notice that the spin-1/2 sector of the theory enters our expressions through one dimensional geometrical contours (Euclidean "worldlines"), while all the dynamics is contained in the expectation values $\left.\left.\left.\langle\cdot \cdot\rangle_{A}^{c o n n}=<\cdot \cdot\right\rangle_{A}-<\cdot\right\rangle_{A}<\cdot\right\rangle_{A}$ of Wilson line operators?

\footnotetext{
${ }^{3}$ We denote by $A$ the gauge field expanded in the Lie algebra. Also, our compressed notation implies that an expectation value in the gauge field sector includes gauge fixing terms, ghost integration and, in the generic case, contribution from the Dirac determinant.
} 
The spacetime setting of the worldline approach affords us to designate points $z_{1}$ and $z_{2}$, on the respective contours $x^{I}(\tau)$ and $x^{I I}(\tau)$, of closest approach. Setting $x^{I}\left(s_{1}\right) \equiv$ $z_{1}, x^{I I}\left(s_{2}\right) \equiv z_{2}$ and using the identity $\int_{0}^{T} \frac{d s}{T} \int d^{4} z \delta[x(s)-z]=1$ we write

$$
\begin{aligned}
\mathcal{M}_{j j^{\prime}}^{i i^{\prime}}=\sum_{C_{x_{1}, y_{1}}^{I}} \sum_{C_{x_{2}, y_{2}}^{I I}} I\left[\dot{x}^{I}\right] I\left[\dot{x}^{I I}\right] \quad & \int_{0}^{T_{1}} \frac{d s_{1}}{T_{1}} \int_{0}^{T_{2}} \frac{d s_{2}}{T_{2}} \int d^{4} z_{1} \delta\left[x^{I}\left(s_{1}\right)-z_{1}\right] \\
& \times \int d^{4} z_{2} \delta\left[x^{I I}\left(s_{2}\right)-z_{2}\right] E\left(C^{I}, C^{I I} ; z_{1}, z_{2}\right)_{j j^{\prime}}^{i i^{\prime}}
\end{aligned}
$$

wheref

$$
\begin{aligned}
& E\left(C^{I}, C^{I I} ; z_{1}, z_{2}\right)_{j j^{\prime}}^{i i^{\prime}}= \\
& <\operatorname{Pexp}\left[i g \int_{0}^{s_{1}} d \tau \dot{x}^{I}(\tau) \cdot A\left(z_{1}-\int_{\tau}^{s_{1}} \dot{x}^{I}\right)+i g \int_{s_{1}}^{T_{1}} d \tau \dot{x}^{I} \cdot A\left(z_{1}+\int_{s_{1}}^{T_{1}} \dot{x}^{I}\right)\right]_{i i^{\prime}} \\
& \quad \times P \exp \left[i g \int_{0}^{s_{2}} d \tau \dot{x}^{I I} \cdot A\left(z_{2}-\int_{\tau}^{s_{2}} \dot{x}^{I I}\right)+i g \int_{s_{2}}^{T_{2}} d \tau \dot{x}^{I I} \cdot A\left(z_{2}+\int_{s_{2}}^{T_{2}} \dot{x}^{I I}\right)\right]_{j j^{\prime}}>_{A}^{\text {conn }} .(5)
\end{aligned}
$$

The above battery of expressions contains the basic formalism of the worldline approach to non-abelian gauge systems with spin-1/2 matter fields pertaining to the four-point process. Our adjustments, from hereon, will refer to the particular situations, i.e. Regge and fixed angle kinematics we intend to study in this and the next section, respectively.

\subsection{Eikonal approximation in the worldline formalism}

Our main applications of the worldline scheme have addressed themselves to the issue of isolating a subsector of a given microscopic theory which can be characterized as "soft". By the latter term we mean a restriction to the study of the physics which is active in the field theory below a given scale $\tilde{\Lambda}$. Clearly, the full microscopic theory contains degrees of freedom associated with higher frequencies. Our strategy, however, is to incorporate them into the definition of the physical quantities entering the theory at scale $\tilde{\Lambda}$ (bare values). For the purposes of this section the aformentioned scale could correspond either to physical mass of the matter field quanta ? (on-mass-shell situation) or to an off-shell mass value.

\footnotetext{
${ }^{4}$ Notice the notational shortcut: $\int_{s}^{T} d \tau \dot{x}(\tau) \rightarrow \int_{s}^{T} \dot{x}$ inside the arguments of the $A$ 's.

${ }^{5}$ We resrtict ourselves to a single physical mass parameter $m$. Adjustments pertaining to different masses could be made at the expense of burdening our analysis with extra formalism that would detract from our main objectives.
} 
The beauty of the worldline casting of the field system is that it effects the isolation of the "soft" subsector in a most efficient and straight forward manner: One instructs the path integral to take into consideration only those paths that are straight lines almost everywhere (allowing, therefore, for the presence of cusps) and sets the Dirac determinant to unity. In physical terms the above specifications imply that matter fields have been dressed to the point that the live, in the considered subsector of the full theory, gauge field exchanges can neither derail them from their propagation paths nor create virtual pairs from the vacuum. Any derailment occurs on a sudden impulse basis and corresponds to the presence of cusps on the propagation contour.

Now, the "soft" subsector has its own UV and IR domains. The latter presumably coincides with that of the full theory, while the former provides anomalous dimensions which induce renormalization group running of physical quantities. Renormalization factors in the subtheory are exclusively associated with the (almost everywhere) straight line configurations and depend solely on the number of cusps that a given contour, relevant to the situation being studied, has. In particular, a (single) straight line of propagation from an initial to a final space-time point signifies negligible momentum transfer to the matter particle. In such a case a wave function renormalization factor is all that is found to be associated with the contour. A cusp, on the other hand, implies a transfer of momentum to the propagating matter particle which occurs on a sudden impulse basis. One is then faced with the task to renormalize the vertex that forms at the derailment point.

The above general comments, offered as a way to provide a first feeling concerning renormalization aspects of the subtheory which has been isolated by our aformentioned stipulations, will receive quantitative treatment throughout our analysis. For the moment, let us consider the case of a four-point process involving two straight line paths $L^{I}$ and $L^{I I}$ characterized by four-velocities $u_{1}\left(=\dot{x}^{I}\right)$ and $u_{2}\left(=\dot{x}^{I I}\right)$, respectively. Let the closest point of approach be $|z|\left(=\left|z_{1}-z_{2}\right|\right)<\sigma$, where $\sigma$ represents the (finite) length of the worldline paths and is of the order of the inverse off-mass-shell momentum scale. The exchanged gauge field quanta are assumed to be too soft to derail the two matter particles, propagating along $L^{I}$ and $L^{I I}$, in any appreciable way. This situation is precisely what one encounters when studying high energy forward scattering in the eikonal approximation and is depicted 
in Fig.2a. We have already considered the applicability of this scheme to QED as well as a linear version of quantum gravity [6]. As already stated our concern, in this paper, pertains to non-abelian gauge systems (QCD).

In the eikonal frame of description, as specified above, Eq (5) reads (we set $|z|=z$ for economy)

$$
\begin{aligned}
E\left(L^{I}, L^{I I} ; z\right)_{j j^{\prime}}^{i i^{\prime}}= & <\operatorname{Pexp}\left[i g \int_{-\sigma}^{+\sigma} d \tau u_{1} \cdot A\left(\tau u_{1}\right)\right]_{i i^{\prime}} \\
& \times \operatorname{Pexp}\left[i g \int_{-\sigma}^{+\sigma} d \tau u_{2} \cdot A\left(z+\tau u_{2}\right)\right]_{j j^{\prime}}>_{A}^{\text {conn }}
\end{aligned}
$$

The above expression furnishes, in the worldline scheme, the dynamical factor which enters the amplitude, cf. Eq (4), and will serve as the central piece of attention in our subsequent analysis.

\subsection{Off mass shell IR regularization and renormalization issues}

It is clear that the quantity given by (6) is UV safe as long as $\frac{1}{z}<\infty$ whereas it is protected from IR singularities if $\sigma<\infty$. The latter specification corresponds to an off mass shell description of the matter particle as the finite length of its propagation contour cuts off all gauge field modes with momentum less than $1 / \sigma$ which participate in its full, on mass shell description.

We shall proceed to investigate the behaviour of the dynamical factor $E_{j j^{\prime}}^{i i^{\prime}}$ by controlling the IR divergencies through line contours of finite length and, following Korchemsky [26], consider UV implications as $z \rightarrow 0$. The relevant singularity is designated as 'cross singularity' since it arises at a point where the two worldlines cross each other. Employing dimensional regularization for controlling this cross UV divergence, a mass scale $\mu$ is introduced which, for finite $z$ now, implicates a renormalization group running up to the scale $1 / z$. Accordingly, our primary task is to determine the anomalous dimensions which will enter the renormalization group equation.

Given the presence of path ordered exponentials, entering on account of the non-abelian setting, our only option is to proceed perturbatively. We write

\footnotetext{
${ }^{6}$ We are referring strictly to exchanges between the two lines (connected four-point function).
} 


$$
E_{j j^{\prime}}^{i i^{\prime}}=(i g)^{2} t_{i i^{\prime}}^{\alpha} t_{j j^{\prime}}^{\alpha} \int_{-\sigma}^{+\sigma} d \tau \int_{-\sigma}^{+\sigma} d \tau^{\prime} u_{1} \cdot u_{2} D\left(\left|u_{1} \tau-u_{2} \tau^{\prime}\right|\right)+\mathcal{O}\left(g^{4}\right)
$$

where, employing the Feynman gauge, one has

$$
D(|x|)=\mu^{4-D} \int \frac{d^{D} k}{(2 \pi)^{D}} e^{-i k \cdot x} \frac{1}{k^{2}}=\frac{\mu^{4-D}}{4 \pi^{D / 2}} \Gamma\left(\frac{D}{2}-1\right) \frac{1}{|x|^{D-2}} .
$$

We easily determine that

$$
\int_{-\sigma}^{+\sigma} d \tau \int_{-\sigma}^{+\sigma} d \tau^{\prime} u_{1} \cdot u_{2} D\left(\left|u_{1} \tau-u_{2} \tau^{\prime}\right|\right)=\frac{1}{4 \pi^{2}}\left(\frac{\mu^{2}}{\tilde{\lambda}^{2}} \pi\right)^{\epsilon} \frac{1}{2 \epsilon} f_{4-2 \epsilon}(w)
$$

where $w \equiv u_{1} \cdot u_{2}, \tilde{\lambda} \equiv 1 / \sigma, \epsilon=4-D(>0)$ and

$$
\begin{aligned}
f_{4-2 \epsilon}= & 4 w\left[\sqrt{\pi} \Gamma\left(\frac{1}{2}-\epsilon\right)(1-w)^{\epsilon-\frac{1}{2}}-\frac{1}{2}(1+w)^{\epsilon} F\left(1,1-\epsilon ; \frac{3}{2}-\epsilon ; \frac{1-w}{2}\right)\right. \\
& \left.-\frac{1}{2}(1-w)^{\epsilon} F\left(1,1-\epsilon ; \frac{3}{2}-\epsilon ; \frac{1+w}{2}\right)\right] .
\end{aligned}
$$

Setting $w=\cos \theta$ we obtain, in the limit $\epsilon \rightarrow 0$,

$$
f_{4}(\theta)=2 \pi \cot \theta
$$

and in Minkowski space $(\theta \rightarrow-i \gamma)$

$$
f_{4}(\gamma)=2 \pi i \operatorname{coth} \gamma
$$

Subtracting the pole term in (7), using the $\overline{\mathrm{MS}}$ scheme, we write

$$
\left(E_{1}\right)_{j j^{\prime}}^{i i^{\prime}}=(i g)^{2} t_{i i^{\prime}}^{\alpha} t_{j j^{\prime}}^{\alpha} \frac{1}{4 \pi^{2}}\left[\ln \left(\frac{\mu^{2}}{\tilde{\lambda}^{2}}\right)+h(\gamma)\right] i \pi \operatorname{coth} \gamma
$$

where

$$
h(\gamma) \equiv \frac{1}{i \pi \operatorname{coth} \gamma} \lim _{\epsilon \rightarrow 0} \frac{1}{2 \epsilon}\left[f_{4-2 \epsilon}(\gamma)-f_{4}(\gamma)\right]
$$

In the limit $\gamma \sim s / m^{2} \rightarrow \infty$, where $m$ stands for the single, according to our agreement, fermion mass scale one obtains

$$
h(\gamma)=\ln \left(s / m^{2}\right)
$$

It is important to realize that $h(\gamma)$ has entered our analysis on account of the off mass shell procedure we are currently pursuing. 
Taking into consideration the fact that the $t$-matrices are in the fundamental representation we finally get

$$
\left(E_{1}\right)_{j j^{\prime}}^{i i^{\prime}}=c_{11} \delta_{i i^{\prime}} \delta_{j j^{\prime}}+c_{12} \delta_{i j^{\prime}} \delta_{j i^{\prime}}
$$

where

$$
c_{11}=\frac{\alpha_{s}}{2 \pi} \ln \left(M^{2} / \tilde{\lambda}^{2}\right) \frac{1}{N} i \pi \operatorname{coth} \gamma+\mathcal{O}\left(\alpha_{s}^{2}\right)
$$

and

$$
c_{12}=-\frac{\alpha_{s}}{2 \pi} \ln \left(M^{2} / \tilde{\lambda}^{2}\right) i \pi \operatorname{coth} \gamma+\mathcal{O}\left(\alpha_{s}^{2}\right)
$$

with $M^{2} \equiv \mu^{2} e^{h(\gamma)}$.

\subsection{Reggeization of exchanged gluons}

The UV structure that has emerged from our considerations in the previous subsection has produced, to $\mathcal{O}\left(\alpha_{s}^{2}\right)$, anomalous dimensions of the form

$$
\Gamma_{\text {cross }}=\frac{\alpha_{s}}{\pi}\left(-\frac{i \pi}{N} \operatorname{coth} \gamma, i \pi \operatorname{coth} \gamma\right)
$$

As Brandt et al. [20] have already pointed out, albeit within the context of a Wilson loop analysis (see next subsection), under renormalization group running quantitity $\left(E_{1}\right)_{j j^{\prime}}^{i i^{\prime}}$ mixes with

$$
\begin{aligned}
\left(E_{2}\right)_{j j^{\prime}}^{i i^{\prime}}= & <\operatorname{Pexp}\left[i g \int_{-\infty}^{0} d \tau u_{1} \cdot A\left(\tau u_{1}\right)+i g \int_{0}^{+\infty} d \tau u_{2} \cdot A\left(\tau u_{2}\right)\right]_{i j^{\prime}} \\
& \times \operatorname{Pexp}\left[i g \int_{-\infty}^{0} d \tau u_{2} \cdot A\left(\tau u_{2}\right)+i g \int_{0}^{+\infty} d \tau u_{1} \cdot A\left(\tau u_{1}\right)\right]_{j i^{\prime}}>_{A}-\delta_{i j^{\prime}} \delta_{j i^{\prime}} .
\end{aligned}
$$

Notice that the worldline configuration entering the above expression consists of two independent cusped lines (see Fig.2b). The two cusps face each other and constitute sources of bremsstrahlung emission. We shall have more comments to make on this matter in the next subsection. For now let us turn our attention to renormalization issues associated with this quantity which are, this time, indigenous on account of the momentum transfer that accompanies each cusp and which can be unboundedly large.

For computational convenience we choose to simulate on mass shell regularization methodology for confronting the IR divergences, introducing for this purpose a (small) mass $\lambda \sim$ $\tilde{\lambda} \sim \frac{1}{\sigma}$ for the gauge field quanta. This simplifies the computation, which now involves four 
Wilson lines, as we are able to assign unit magnitude to each four-velocity. Consistency with our off mass shell IR regularization can, on the other hand, be achieved by employing the energy dependent renormalization scale $M^{2} \equiv \mu^{2} e^{h(\gamma)}$ identified in the previous subsection.

We readily determine, after making the necessary readjustment in order to attain a result compatible with the $\overline{\mathrm{MS}}$ subtraction scheme,

$$
\begin{aligned}
& \left(E_{2}\right)_{j j^{\prime}}^{i i^{\prime}}= \\
& 2(i g)^{2} c_{F} \delta_{i j^{\prime}} \delta_{j i^{\prime}}\left[\int_{0}^{\infty} d \tau \int_{0}^{\infty} d \tau^{\prime} D\left(\left|u \tau-u \tau^{\prime}\right| ; M\right)+\int_{0}^{\infty} d \tau \int_{0}^{\infty} d \tau^{\prime} D\left(\left|u_{1} \tau+u_{2} \tau^{\prime}\right| ; M\right) u_{1} \cdot u_{2}\right] \\
& +2(i g)^{2} t_{i j^{\prime}}^{\alpha} t_{j i^{\prime}}^{\alpha}\left[\int_{0}^{\infty} d \tau \int_{0}^{\infty} d \tau^{\prime} D\left(\left|u \tau+u \tau^{\prime}\right| ; M\right)+\int_{0}^{\infty} d \tau \int_{0}^{\infty} d \tau^{\prime} D\left(\left|u_{1} \tau-u_{2} \tau^{\prime}\right| ; M\right) u_{1} \cdot u_{2}\right] \\
& +\mathcal{O}\left(g^{4}\right),
\end{aligned}
$$

where $u$ stands, where it appears, generically for $u_{1}$ and $u_{2}$ and

$$
D(|x| ; M)=M^{4-D} \int \frac{d^{D} k}{(2 \pi)^{D}} e^{-i k \cdot x} \frac{1}{k^{2}+\lambda^{2}} .
$$

Once more we emphasize that the seemingly on mass shell regularization implied by the above formula is adjusted to the off mass shell strategy, that we have been adhering to, via the use of the energy-dependent mass $M$ as our renormalization point.

In the limit of asymptotically high energies, we obtain, in Minkowski space,

$$
\left(E_{2}\right)_{j j^{\prime}}^{i i^{\prime}}=\delta_{i i^{\prime}} \delta_{j j^{\prime}} c_{21}+\delta_{i j^{\prime}} \delta_{j i^{\prime}} c_{22}
$$

where

$$
c_{21}=-\frac{\alpha_{s}}{2 \pi} \ln \left(M^{2} / \lambda^{2}\right)[\gamma \operatorname{coth} \gamma+i \pi \operatorname{coth} \gamma]+\mathcal{O}\left(\alpha_{s}^{2}\right)
$$

and

$$
c_{22}=-\frac{\alpha_{s}}{2 \pi} \ln \left(M^{2} / \lambda^{2}\right)\left[N(\operatorname{coth} \gamma-1)-\frac{i \pi}{N} \operatorname{coth} \gamma\right]+\mathcal{O}\left(\alpha_{s}^{2}\right)
$$

From the above relations we read the anomalous dimensions associated with the "pair cusp' configuration as follows

$$
\Gamma_{\text {pair cusp }}=\frac{\alpha_{s}}{\pi}\left(-\gamma \operatorname{coth} \gamma+1+i \pi \operatorname{coth} \gamma, N(\operatorname{coth} \gamma-1)-\frac{i \pi}{N} \operatorname{coth} \gamma\right)
$$

Combining the above result with that of Eq. (19) we obtain the following $2 \times 2$ anomalous dimension matrix

$$
\left(\Gamma_{a b}\right)=\frac{\alpha_{s}}{\pi}\left(\begin{array}{ll}
-\frac{i \pi}{N} \operatorname{coth} \gamma & i \pi \operatorname{coth} \gamma \\
-\gamma \operatorname{coth} \gamma+1+i \pi \operatorname{coth} \gamma & N(\operatorname{coth} \gamma-1)-\frac{i \pi}{N} \operatorname{coth} \gamma
\end{array}\right)
$$


which governs the running of the quantities $E_{1}$ and $E_{2}$ under the renormalization group equation. The generic form of the latter is

$$
\left(M \frac{\partial}{\partial M}+\beta(g) \frac{\partial}{\partial g}\right) E_{a}=\Gamma_{a b}(\gamma, g) E_{b}
$$

At this point we have established full contact with Korchemsky's operator-based analysis. Following Ref. [26], we introduce amplitudes $T_{+}$which enter expressions for the singlet and octet components of the invariant amplitude whose LLA form is given, according to the renormalization group equation (28), by

$$
T_{\underline{+}}=\frac{t}{\Gamma_{+}} \int d^{2} z e^{-i \vec{z} \cdot \vec{q}} \exp \left[-\Gamma_{+} \int_{\lambda}^{\frac{1}{z}} \frac{d \tau}{\tau} \frac{\alpha_{s}(\tau)}{\pi}\right],
$$

where $\Gamma_{+_{-}}$are the eigenvalues of the matrix $\Gamma_{a b}$ and $\bar{z}^{2}=z^{2} e^{-h(\gamma)}$ whose asymtotic expressions read $\Gamma_{+}=N \ln \frac{s}{M^{2}}, \Gamma_{-}=\pi^{2} \frac{N^{2}-1}{N^{3}} \frac{1}{\ln \frac{s}{M^{2}}}$.

Given that

$$
\begin{aligned}
\int_{\lambda}^{\frac{1}{z}} \frac{d \tau}{\tau} \frac{\alpha_{s}}{\pi} & =\frac{2}{\beta_{o}} \ln \left[\frac{\ln \left(\frac{1}{\bar{z} \Lambda_{Q C D}^{2}}\right.}{\ln \left(\frac{\lambda^{2}}{\Lambda_{Q C D}^{2}}\right.}\right] \\
& =\frac{2}{\beta_{o}} \ln \left[1+\frac{\beta_{o}}{4 \pi} \alpha_{s} \ln \frac{1}{z^{2} \hat{\lambda}^{2}}\right]
\end{aligned}
$$

where $\hat{\lambda}^{2}=\lambda^{2} \frac{m^{2}}{s}$, we obtain

$$
T_{+}=2 \alpha_{s} \exp \left[-\frac{\alpha_{s}}{2 \pi} \Gamma_{+} \ln \frac{(-t)}{\hat{\lambda}^{2}}\right] \Gamma\left(1+\frac{\alpha_{s}}{2 \pi} \Gamma_{+}\right) / \Gamma\left(1-\frac{\alpha_{s}}{2 \pi} \Gamma_{+}\right) .
$$

The above result contributes to the octet part of the forward amplitude through an expression which explicitly exhibits the reggeization of the exchanged gluons:

$$
T_{L L} \sim\left(\frac{s}{M^{2}}\right)^{\beta}
$$

where $L L$ stands for 'leading logarithm' and

$$
\beta=-\frac{\alpha_{s}}{2 \pi} N \ln \frac{-t}{\hat{\lambda}^{2}}=-\frac{\alpha_{s}}{2 \pi}\left[\ln \frac{-t}{\lambda^{2}}+\ln \frac{s}{m^{2}}\right]
$$

implying the more suggestive form

$$
T_{L L} \sim\left(\frac{s}{m^{2}}\right)^{\alpha(t)} e^{-\frac{\alpha_{s}}{2 \pi} N \ln ^{2} \frac{s}{m^{2}}}
$$


One reads, from the above expression, the Regge trajectory as $\alpha(t)=-\frac{\alpha_{s}}{2 \pi} N \ln \frac{-t}{m^{2}}$.

A notable difference is recorded with respect to gluon reggeization results obtained by other, non-worldline, methodologies, namely the appearance of the exponential factor $e^{-\frac{\alpha_{s}}{2 \pi} N n^{2} \frac{s}{m^{2}}}$. This is directly attributable to our use of an off mass shell IR regularization strategy, as opposed to the on mass shell practice employed in other works. Comparing, e.g., with Ref. 29, where the anomalous dimension structure for quark scattering was first investigated, one observes full agreement with our results. In particular, modulo a reverse designation of the + and - components, the eigenvalues of the anomalous dimension matrix coincide.

Our final expression for the amplitude, with its damping factor, presents an inetrest of its own in connection with unitarity requirements. The general guidelines for effecting unitarization in the amplitudes for high energy processes, in the conventional framework of Feynman diagrams, have been elegantly discussed by Cheng and Wu (Ref. 1, last chapter). On a more concrete basis, systematic attempts to deal with unitarization of the quark-quark scattering amplitude have been pursued by Lipatov [29-31] who has confronted the unitarity issue, within the context of multi-Regge kinematics, in terms of an eikonal-based expression for the S-matrix in the impact parameter space.

Even though we shall not enter unitarization issues in the present paper, it is worth making some comparisons with more recent studies, [32-34], which employ similar methods with ours to arrive at a description of high-energy scattering in QCD in terms of an effective two-dimensional field theory. Focusing on unitarity and gauge invariance, the above authors have recognized the importance of facilitating the derivation of such effective actions by employing Wilson, straight-line contour integrals. In our approach, of course, Wilson line operators are an integral part of the very formulation of the field system and carry, in fact, its dynamics. The main difference is that whereas we rely on Wilson lines of finite extent, in the work of Refs. 32-34 lines of infinite extent, equivalently Wilson loops, are employed. The resulting off mass shell treatment of IR divergences in our case offers a different perspective in that it differentiates what is 'soft', but observable, and what is 'infrared' and attributable to unobservable, with respect to the scattering dynamics, modes (wavelengths $\geq \frac{1}{\sigma}$ ). The latter contribute exclusively to the non-perturbative dynamics of QCD and we surmise that 
their exclusion from our considerations is precisely the reason for the emergence of the damping factor in (34). It is certainly of great interest to identify the connection between the conventional multi-production, in the $s$-channel, approach to unitarity and the damping factor which makes its appearance in our work.

\subsection{Miscellaneous remarks}

A number of observations and/or remarks stemming from our worldline approach to nonabelian scattering in the Regge limit and which might be of some interest will be presented in this subsection.

To begin, we wish to consider possible connections with past work centered around Wilson loops $[20,21]$. To this end, let us focus on the on-mass shell case where the matter particle worldlines extend to infinity. In a Euclidean space-time background two such lines join at infinity, thereby forming closed paths. The corresponding closed loop configurations for the 'crossed' and 'pair-cusp' cases are depicted, respectively, in figures 3a,b. It follows that there is a direct correspondence between studies performed in relation to Wilson loops and dynamical considerations taking place within the worldline approach. The fact, on the other hand, that in our case Wilson loops/lines enter the formulation of the field system directly and not as formally introduced objects, underlines their role as fundamental ingredients of the field theoretical description per se. One immediate aftermath of this occurence has already been witnessed in the present work, namely the ability to utilize off-mass shell properties.

A second point of interest concerns the relevance of the paired-cusp configuration which entered the renormalization group study of the forward amplitude. Cusps on Wilson loops are associated with bremsstrahlung radiation [19]. In a diagrammatic context, on the other hand, such a situation would reveal itself if we were to make a 'horizontal' unitarity-type cut accross the $t$-channel. It is of interest to note that the $s-t$ interplay, which is quintessential to unitarity enforcement at high energies [1], seems to be in a one-to-one correspondence with the operator mixing induced by the renormalization group. We feel that this is an issue that merits further study.

Turning our attention to QED, let us observe that the abelian nature of the theory allows us to treat the expectation value of the ordinary Wilson exponential as the exponential of 
the correlator. This leads us directly to the eikonal form for the dynamical factor $\square E$, which, for off mass shell IR regularization, reads

$$
E \sim 1-e^{i \chi_{o}}, \quad i \chi_{o}=-\frac{\alpha}{\pi}(i \pi \operatorname{coth} \gamma) \ln \frac{1}{z^{2} \hat{\lambda}^{2}}
$$

where $\hat{\lambda}^{2} \equiv \tilde{\lambda}^{2} e^{-h(\gamma)}$.

For the amplitude one obtains

$$
A \sim \int d^{2} z e^{i \vec{q} \cdot \vec{z}} e^{i \chi_{o}}=4 \pi i \alpha \frac{\operatorname{coth} \gamma}{t}\left(-\frac{t}{\hat{\lambda}^{2}}\right)^{-i \alpha \operatorname{coth} \gamma} \frac{\Gamma(1+i \alpha \operatorname{coth} \gamma)}{\Gamma(1-i \alpha \operatorname{coth} \gamma)}
$$

whose asymptotic form, as $s / m^{2} \rightarrow \infty$, reads

$$
A \sim 4 \pi i \alpha \frac{1}{t}\left(-\frac{t}{\hat{\lambda}^{2}}\right)^{-i \alpha} \frac{\Gamma(1+i \alpha)}{\Gamma(1-i \alpha)}\left(\frac{s}{m^{2}}\right)^{-i \alpha} .
$$

One last reference to QED, which pertains to a 'visual' suggestion facilitated through its worldine casting, is the following. Suppose that in a basically forward process one also allows for the observations of "soft" photons, i.e. photons which do not exceed a given energy scale $\tilde{\Lambda}$. An $s$-channel study for this process can be suggested, in a space-time setting, by extracting a 'region' of radius $T \sim \frac{1}{\hat{\Lambda}}$ centered around the point of closest approach, see Fig 4a. Upon cutting, in the Feynman diagrammatic context, along the $t$-direction we obtain the cross sectional profile of an inclusive process involving 'soft' photon emission, as per our requirement, see Fig 4b. The difference brought about in (35) corresponds to a modification of the eikonal function of the form $\chi_{o} \rightarrow \tilde{\chi}_{o}$ which, we speculate, that for large enough $T$ is consistent with the presence of a diffraction pattern in the forward direction. In view of experimental observations [35] which report a notable excess of soft photons in the forward direction, our aformentioned speculation might be worth to consider further.

\section{High-energy, non-abelian scattering at fixed angles}

Our considerations in this Section will be extended to the case where the four-velocities entering each of the four branches in (5) are different from one another. In particular, we

\footnotetext{
${ }^{7}$ Aside from the obvious fact that no group indices are involved here, there is no need for putting a subscript on $E$ as exponentiation is now automatically obtained and a renormalization group running is no longer required.
} 
set $\dot{x}^{I}=u_{1}$ in $\left[0, s_{1}\right], \dot{x}^{I}=u_{1}^{\prime}$ in $\left[s_{1}, T_{1}\right], \dot{x}^{I I}=u_{2}$ in $\left[0, s_{2}\right]$ and $\dot{x}^{I I}=u_{2}^{\prime}$ in $\left[s_{2}, T_{2}\right]$, see Fig. 5a. For simplicity, we shall work with disconnected correlation functions which we denote by $\left(W_{1}\right)_{j j^{\prime}}^{i i^{\prime}}$, where the subscript ' 1 ' pertains to the crossed configuration. (Later we shall use ' 2 ' as the subscript for a pair-cusped configuration which mixes in, under renormalization group running.)

Non-abelian group complications force us to define the following invariant quantities, see, e.g., $\operatorname{Ref}[26]$,

$$
W_{1}^{(a)} \equiv<\operatorname{tr} P_{I} \operatorname{tr} P_{I I}>_{A}=\delta_{i i^{\prime}} \delta_{j j^{\prime}}\left(W_{1}\right)_{j j^{\prime}}^{i i^{\prime}}
$$

and

$$
W_{1}^{(b)} \equiv<\operatorname{tr}\left(P_{I} P_{I I}>_{A}=\delta_{i j^{\prime}} \delta_{j i^{\prime}}\left(W_{1}\right)_{j j^{\prime}}^{i i^{\prime}}\right.
$$

where $P_{I}$ denotes the line configuration parametrized by $\dot{x}^{I}$ and $P_{I I}$ the one parametrized by $\dot{x}^{I I}$.

It follows that

$$
\left(W_{1}\right)_{j j^{\prime}}^{i i^{\prime}}=\frac{N W_{1}^{(a)}-W_{1}^{(b)}}{N\left(N^{2}-1\right)} \delta_{i i^{\prime}} \delta_{j j^{\prime}}+\frac{N W_{1}^{(b)}-W_{1}^{(a)}}{N\left(N^{2}-1\right)} \delta_{i j^{\prime}} \delta_{j i^{\prime}} .
$$

A final introductory note pertains to our kinematical parametrization. We make the following choice [36] for the particle momenta on each of the four branches (consistent, of course, with an over all four-momentum conservation):

$$
\begin{aligned}
& p_{1}=\left(\sqrt{Q^{2}+M^{2}}, 0,0, Q\right), \quad p_{2}=\left(\sqrt{Q^{2}+M^{2}}, 0,0,-Q\right) \\
& p_{1}^{\prime}=\left(\sqrt{Q^{2}+M^{2}}, 0, Q \sin \theta, Q \cos \theta\right), \quad p_{2}^{\prime}=\left(\sqrt{Q^{2}+M^{2}}, 0,-Q \sin \theta,-Q \cos \theta\right)
\end{aligned}
$$

which, in turn, parametrizes the $s$ and $t$ variables as follows

$$
s=\left(p_{1}+p_{2}\right)^{2}=4\left(Q^{2}+M^{2}\right)
$$

and

$$
t=\left(p_{1}-p_{1}^{\prime}\right)^{2}=-2 Q^{2}(1-\cos \theta)
$$

The limit $s, t \rightarrow \infty$ with $s / t$ fixed will be taken in the sense $Q \rightarrow \infty, \theta$ fixed.

\subsection{Hard-Soft Factorization in the Subtheory}


Unlike the forward scattering case we now have to face a situation where a sizeable momentum transfer is involved in the considered process which, according to our parametrization, is of order $Q$. The latter sets the scale beyond which no corresponding degree of freedom explicitly enters our analysis, hence it is wise to "dress" our quantities at least down to that scale. Within the remaining 'live' sector of the theory we introduce an intermediate scale $\Lambda$ which separates soft from hard gluons and whose arbitrariness will naturally induce a renormalization group running in the subtheory. Moreover, we shall place the matter particles on-shell, i.e. we shall employ worldlines of infinite extent, thereby regulating the IR divergencies through a small gluon mass $\lambda$.

As $\Lambda$ stands between $Q$ and $\lambda$, what one calls "soft" and what "hard" is relative. For example, if one were to play with $\Lambda$, say lower it, then gluons that were originally debited to the soft transfer to the hard group. The opposite happens, of course, when the value of $\Lambda$ is raised. The factorized relation for the invariant quantities $W_{1}^{(a, b)}$ reads

$$
W_{1}^{(a, b)}=\left(W_{1}^{(a, b)}\right)_{S O F T}\left(W_{1}^{(a, b)}\right)_{H A R D}+\mathcal{O}\left(\frac{1}{\Lambda^{2}}\right)
$$

The arbitrariness of the dividing scale calls for a renormalization group running which will lead to our final expression for $W_{1}^{(a, b)}$ and, by extension, for the amplitude. The manner in which this strategy will be effected is the subject of concern in the present subsection.

Let us start by recalling our discussion in subsection 2.2 according to which, given the (cusped) line configurations for each of the two colliding particles, soft gluons correspond to what is emitted or absorbed by the straight line segments (no impulse approximation). In this soft sector of the full theory one determines anomalous dimensions associated with its own high energy domain ${ }^{\$}$. For a given $\Lambda$, one can induce a renormalization group running of $\left(W_{1}\right)_{S O F T}$ from $\lambda$ to $\Lambda$.

With the above observation in place, our next remark is that $\left(W_{1}^{(a, b)}\right)_{S O F T}$ exhibits a dependence on $Q$ through the angle $\theta$ formed at a given cusp, e.g. $\cos \theta=u_{1} \cdot u_{1}^{\prime}$. We

\footnotetext{
${ }^{8}$ This is not a novel idea. For example, in the Bloch-Nordsieck approximation [37], which describes the soft limit of QED, one discovers anomalous dimensions [38] which lead to the proper form of the full fermion propagator in the IR. The point is that, from the perspective of the IR cutoff $\lambda$, the upper momentum scale $Q$ appears as infinite.
} 
thereby write

$$
\frac{d}{d \ln Q^{2}} \ln W_{1}^{(a, b)}=\frac{d}{d \ln Q^{2}} \ln \left(W_{1}^{(a, b)}\right)_{S O F T}+\frac{d}{d \ln Q^{2}} \ln \left(W_{1}^{(a, b)}\right)_{H A R D} .
$$

Now, the renormalization group equation for the quantity $W_{1}^{(a, b)}$ as a whole, which runs in the interval $[\lambda, Q]$, reads

$$
\left(\mu \frac{\partial}{\partial \mu}+\beta(g) \frac{\partial}{\partial g}\right) \frac{d}{\ln Q^{2}} \ln W_{1}^{(a, b)}=0
$$

and expresses independence from the scale that separates soft from hard physics within the considered subtheory. (We have used $\mu$ to represent $\Lambda$ in order to underline the fact that we are letting the latter scale to run.)

Factorization, then, gives

$$
\begin{aligned}
& \left(\mu \frac{\partial}{\partial \mu}+\beta \frac{\partial}{\partial g}\right) \frac{d}{d \ln Q^{2}} \ln \left(W_{1}^{(a, b)}\right)_{H A R D} \\
& \quad=-\left(\mu \frac{\partial}{\partial \mu}+\beta \frac{\partial}{\partial g}\right) \frac{d}{d \ln Q^{2}} \ln \left(W_{1}^{(a, b)}\right)_{S O F T}
\end{aligned}
$$

But, provided we find the anomalous dimensions associated with the soft factor, the expression on the right enters the renormalization group equation discussed above along with a term of the form: (anomalous dimensions) $\times\left(W_{1}^{(a, b)}\right)_{S O F T}$. Therefore, $W_{1}^{(a, b)}$ can be determined via a two-step procedure which first addresses itself to its soft and second to its hard component.

In the next subsection we shall carry out perturbative calculations pertaining to the soft part which will lead to the determination, to order $\alpha_{s}$, of the anomalous dimension matrix.

\subsection{Perturbative calculations in the soft sector}

We begin our considerations surrounding the soft part of the amplitude by displaying its perturbative expression, to $\mathcal{O}\left(g^{2}\right)$, which reads

$$
\begin{aligned}
{\left[\left(W_{1}\right)_{S O F T}\right]_{j j^{\prime}}^{i i^{\prime}} } & =\delta_{i i^{\prime}} \delta_{j j^{\prime}}+(i g)^{2} c_{F} \delta_{i i^{\prime}} \delta_{j j^{\prime}}\left\{2 \int_{0}^{\infty} d \tau \int_{0}^{\infty} d \tau^{\prime} D\left(\left|\tau u_{1}-\tau^{\prime} u_{1}\right|\right)\right. \\
+ & \left.\int_{0}^{\infty} d \tau \int_{0}^{\infty} d \tau^{\prime} u_{1} \cdot u_{1}^{\prime} D\left(\left|\tau u_{1}+\tau^{\prime} u_{1}^{\prime}\right|\right)+\int_{0}^{\infty} d \tau \int_{0}^{\infty} d \tau^{\prime} u_{2} \cdot u_{2}^{\prime} D\left(\left|\tau u_{2}+\tau^{\prime} u_{2}^{\prime}\right|\right)\right\} \\
+ & (i g)^{2} t_{i i^{\prime}}^{a} t_{j j^{\prime}}^{a}\left\{\int_{0}^{\infty} d \tau \int_{0}^{\infty} d \tau^{\prime} D\left(\left|\tau u_{i}-\tau^{\prime} u_{2}\right|\right)+\int_{0}^{\infty} d \tau \int_{0}^{\infty} d \tau^{\prime} D\left(\left|\tau u_{1}^{\prime}+\tau^{\prime} u_{2}\right|\right)\right. \\
& \left.+\int_{0}^{\infty} d \tau \int_{0}^{\infty} d \tau^{\prime} u_{1} \cdot u_{2}^{\prime} D\left(\left|\tau u_{1}+\tau^{\prime} u_{2}^{\prime}\right|\right)+\int_{0}^{\infty} d \tau \int_{0}^{\infty} d \tau^{\prime} u_{1}^{\prime} \cdot u_{2}^{\prime} D\left(\left|\tau u_{1}^{\prime}-\tau^{\prime} u_{2}^{\prime}\right|\right)\right\} \\
& +\mathcal{O}\left(g^{4}\right)
\end{aligned}
$$


with $D(|x|)$ given by Eq. (22) (we have suppressed the $\mu$ argument for simplicity).

We determine

$$
\int_{0}^{\infty} d \tau \int_{0}^{\infty} d \tau^{\prime} D\left(\left|\tau u_{1}+\tau^{\prime} u_{2}\right|\right)=\frac{1}{(4 \pi)^{D / 2}}\left(\frac{\mu}{\lambda}\right)^{4-D} 2 \Gamma\left(2-\frac{D}{2}\right) \frac{1}{\sqrt{1-w^{2}}} \operatorname{arctg} \frac{\sqrt{1-w^{2}}}{w}
$$

where $w \equiv u_{1} \cdot u_{2}$ and

$$
\int_{0}^{\infty} d \tau \int_{0}^{\infty} d \tau^{\prime} D\left(\left|\tau u_{1}-\tau^{\prime} u_{2}\right|\right)=\frac{1}{(4 \pi)^{D / 2}}\left(\frac{\mu}{\lambda}\right)^{4-D} 2 \Gamma\left(2-\frac{D}{2}\right) \frac{1}{\sqrt{1-w^{2}}}\left[\pi-\operatorname{arctg} \frac{\sqrt{1-w^{2}}}{w}\right] .
$$

The above relations together with (9) give, upon transcription to Minkowski space,

$$
\begin{aligned}
{\left[\left(W_{1}\right)_{S O F T}\right]_{j j^{\prime}}^{i i^{\prime}} } & =\delta_{i i^{\prime}} \delta_{j j^{\prime}}-\frac{g^{2}}{4 \pi^{2}}\left(\frac{\mu^{2}}{\lambda^{2}} \pi\right)^{\epsilon} \frac{\Gamma(1+\epsilon)}{\epsilon}\left\{c_{F} \delta_{i i^{\prime}} \delta_{j j^{\prime}}\left(\gamma_{11^{\prime}} \operatorname{coth} \gamma_{11^{\prime}}-1\right)\right. \\
& +t_{i i^{\prime}}^{a} t_{j j^{\prime}}^{a}\left[\left(i \pi-\gamma_{12} \operatorname{coth} \gamma_{12}+\gamma_{12^{\prime}} \operatorname{coth} \gamma_{12^{\prime}}\right]\right\}+\mathcal{O}\left(g^{4}\right)
\end{aligned}
$$

where $\operatorname{coth} \gamma_{i j}=\frac{1}{m^{2}} p_{i} \cdot p_{j}$ and where we have taken into account that $\gamma_{12}=\gamma_{1^{\prime} 2^{\prime}}, \gamma_{12^{\prime}}=\gamma_{1^{\prime} 2}$, due to momentum conservation.

For the corresponding invariant quantities $W_{1}^{(a, b)}$ we find

$$
\left(W_{1}^{(a)}\right)_{S O F T}=N^{2}\left[1-\frac{\alpha_{s}}{\pi} \ln \left(\frac{\mu}{\lambda}\right) A_{11}\right]-N \frac{\alpha_{s}}{\pi} \ln \left(\frac{\mu}{\lambda}\right) A_{12}+\mathcal{O}\left(\alpha_{s}^{2}\right)
$$

and

$$
\left(W_{1}^{(b)}\right)_{S O F T}=N\left[1-\frac{\alpha_{s}}{\pi} \ln \left(\frac{\mu}{\lambda}\right) A_{11}\right]-N^{2} \frac{\alpha_{s}}{\pi} \ln \left(\frac{\mu}{\lambda}\right) A_{12}+\mathcal{O}\left(\alpha_{s}^{2}\right)
$$

where

$$
A_{11}=2 c_{F}\left(\gamma_{11^{\prime}} \operatorname{coth} \gamma_{11^{\prime}}-1\right)-\frac{1}{N}\left[\left(i \pi-\gamma_{12}\right) \operatorname{coth} \gamma_{12}+\gamma_{12^{\prime}} \operatorname{coth} \gamma_{12^{\prime}}\right]
$$

and

$$
A_{12}=\left(i \pi-\gamma_{12}\right) \operatorname{coth} \gamma_{12}+\gamma_{12^{\prime}} \operatorname{coth} \gamma_{12^{\prime}}
$$

We now bring into play the quantity $\left[\left(W_{2}\right)_{S O F T}\right]_{j j^{\prime}}^{i i^{\prime}}$ given by

$$
\begin{aligned}
{\left[\left(W_{2}\right)_{S O F T}\right]_{j j^{\prime}}^{i i^{\prime}}=<} & \operatorname{Pexp}\left[i g \int_{-\infty}^{0} d \tau u_{1} \cdot A\left(\tau u_{1}\right)+i g \int_{0}^{\infty} d \tau u_{2}^{\prime} \cdot A\left(\tau u_{2}^{\prime}\right)\right]_{i j^{\prime}} \\
& \times \operatorname{Pexp}\left[i g \int_{-\infty}^{0} d \tau u_{2} \cdot A\left(\tau u_{2}\right)+i g \int_{0}^{\infty} d \tau u_{1}^{\prime} \cdot A\left(\tau u_{1}^{\prime}\right)\right]_{j i^{\prime}}>_{A}
\end{aligned}
$$

which mixes with $\left[\left(W_{1}\right)_{S O F T}\right]_{j j^{\prime}}^{i{ }^{\prime}}$ under the renormalization group. The relevant configuration is depicted in Fig. 5b. 
Similar considerations to those that led to (51) now give (in Minkowski space)

$$
\begin{gathered}
{\left[\left(W_{2}\right)_{S O F T}\right]_{j j^{\prime}}^{i i^{\prime}} \quad=\delta_{i j^{\prime}} \delta_{j i^{\prime}}-\frac{g^{2}}{4 \pi^{2}}\left(\frac{\mu^{2}}{\lambda^{2}} \pi\right)^{\epsilon} \frac{\Gamma(1+\epsilon)}{\epsilon}\left\{c_{F} \delta_{i j^{\prime}} \delta_{j i^{\prime}}\left(\gamma_{12^{\prime}} \operatorname{coth} \gamma_{12^{\prime}}-1\right)\right.} \\
+\quad t_{i j^{\prime}}^{a} t_{j i^{\prime}}^{a}\left[\left(i \pi-\gamma_{12} \operatorname{coth} \gamma_{12}+\gamma_{11^{\prime}} \operatorname{coth} \gamma_{11^{\prime}}\right]\right\}+\mathcal{O}\left(g^{4}\right) .
\end{gathered}
$$

The corresponding invariant quantities $\left(W_{2}^{(a, b)}\right)_{S O F T}$ turn out to be

$$
\left(W_{2}^{(a)}\right)_{S O F T}=-N^{2} \frac{\alpha_{s}}{\pi} \ln \left(\frac{\mu}{\lambda}\right) A_{21}+N\left[1-\frac{\alpha_{s}}{\pi} \ln \left(\frac{\mu}{\lambda}\right) A_{22}\right]+\mathcal{O}\left(\alpha_{s}^{2}\right)
$$

and

$$
\left(W_{2}^{(b)}\right)_{S O F T}=-N \frac{\alpha_{s}}{\pi} \ln \left(\frac{\mu}{\lambda}\right) A_{21}+N^{2}\left[1-\frac{\alpha_{s}}{\pi} \ln \left(\frac{\mu}{\lambda}\right) A_{22}\right]+\mathcal{O}\left(\alpha_{s}^{2}\right)
$$

where

$$
A_{21}=\left(i \pi-\gamma_{12}\right) \operatorname{coth} \gamma_{12}+\gamma_{11^{\prime}} \operatorname{coth} \gamma_{11^{\prime}}
$$

and

$$
A_{22}=2 c_{F}\left(\gamma_{12^{\prime}} \operatorname{coth} \gamma_{12^{\prime}}-1\right)-\frac{1}{N}\left[\left(i \pi-\gamma_{12}\right) \operatorname{coth} \gamma_{12}+\gamma_{11^{\prime}} \operatorname{coth} \gamma_{11^{\prime}}\right]
$$

With the above results in place, we are ready to apply the renormalization group analysis for the fixed angle scattering amplitute. The relevant presentation will be given in the next subsection.

\subsection{Renormalization Group running and Sudakov behaviour}

Our perturbative results, to $\mathcal{O}\left(\alpha_{s}\right)$, of the previous subsection lead to a LLA for $\left(W_{1}^{(a, b)}\right)_{S O F T}$ via the renormalization group $(\mathrm{RG})$ equation

$$
\left(\mu \frac{\partial}{\partial \mu}+\beta(g) \frac{\partial}{\partial g}\right) \tilde{W}_{S O F T}^{(i)}=-\frac{\alpha_{s}}{\pi} \tilde{A} \tilde{W}_{S O F T}^{(i)}, \quad i=a, b,
$$

where

$$
\tilde{W}_{S O F T}^{(i)} \equiv\left(\begin{array}{c}
W_{1}^{(i)} \\
W_{2}^{(i)}
\end{array}\right)_{S O F T}
$$

and

$$
\tilde{A}=\left(\begin{array}{cc}
A_{11} & A_{12} \\
A_{21} & A_{22}
\end{array}\right)
$$


The boundary conditions for solving the RG equation are chosen so that no structure is seen at momentum scales below the IR cutoff $\lambda$ :

$$
\left.\tilde{W}_{S O F T}^{(a)}\right|_{\mu=\lambda}=\left(\begin{array}{c}
N^{2} \\
N
\end{array}\right),\left.\quad \tilde{W}_{S O F T}^{(b)}\right|_{\mu=\lambda}=\left(\begin{array}{c}
N \\
N^{2}
\end{array}\right) .
$$

The solution has the general form

$$
\tilde{W}_{S O F T}^{(i)}(\mu / \lambda)=\operatorname{Pexp}\left[-\tilde{A} \int_{\lambda}^{\mu} \frac{d \tau}{\tau} \frac{\alpha_{s}(\tau)}{\pi}\right] \tilde{W}_{S O F T}^{(i)}(1)
$$

In the asymptotic regime of interest we determine

$$
\gamma_{12}=\cosh ^{-1}\left(\frac{s}{2 m^{2}}-1\right)=\cosh ^{-1}\left(\frac{2 Q^{2}}{m^{2}}+1\right) \simeq \ln \left(\frac{2 Q^{2}}{m^{2}}\right)
$$

and, in a similar fashion,

$$
\gamma_{11^{\prime}} \simeq \ln \left(\frac{2 Q^{2}}{m^{2}}\right)+\ln \left(\sin ^{2} \theta\right), \quad \gamma_{12^{\prime}} \simeq \ln \left(\frac{2 Q^{2}}{m^{2}}\right)+\ln \left(\cos ^{2} \theta\right) .
$$

Taking the above into account and following the procedure exhibited in the Appendix we arrive at the asymptotic, as $s,|t| \rightarrow \infty$ at fixed ratio, results

$$
\left(W_{1}^{(a)}\right)_{S O F T} \simeq N^{2} \exp \left[-2 c_{F} \ln \left(\frac{2 Q^{2}}{m^{2}}\right) \int_{\lambda}^{\mu} \frac{d \tau}{\tau} \frac{\alpha_{s}(\tau)}{\pi}\right] 4 c_{F} \ln \left(\frac{2 Q^{2}}{m^{2}}\right) \phi_{1}(\mu / \lambda, \theta)
$$

and

$$
\left(W_{1}^{(b)}\right)_{S O F T} \simeq N \exp \left[-2 c_{F} \ln \left(\frac{2 Q^{2}}{m^{2}}\right) \int_{\lambda}^{\mu} \frac{d \tau}{\tau} \frac{\alpha_{s}(\tau)}{\pi}\right] 4 c_{F} \ln \left(\frac{2 Q^{2}}{m^{2}}\right) \phi_{1}(\mu / \lambda, \theta),
$$

where the function $\phi_{1}(\mu / \lambda, \theta)$ is defined in the Appendix, along with a function $\phi_{2}(\mu / \lambda, \theta)$ which does not appear in the above expressions since its role is inconsequential to our subsequent considerations. Note that $\frac{1}{N}\left(W_{1}^{(a)}\right)_{S O F T} \simeq\left(W_{1}^{(b)}\right)_{S O F T}$.

The resulting expression for the amplitude is

$$
\left[\left(W_{1}\right)_{S O F T}\right]_{j j^{\prime}}^{i i^{\prime}} \simeq\left[\delta_{i i^{\prime}} \delta_{j j^{\prime}}+\frac{N}{N^{2}-1} \delta_{i j^{\prime}} \delta_{j i^{\prime}}\right] F_{S O F T}\left(\frac{Q^{2}}{m^{2}}, \frac{\mu}{\lambda}\right)
$$

where

$$
F_{S O F T}\left(\frac{Q^{2}}{m^{2}}, \frac{\mu}{\lambda}\right)=4 c_{F} \ln \left(\frac{2 Q^{2}}{m^{2}}\right) \exp \left[-2 c_{F} \ln \left(\frac{2 Q^{2}}{m^{2}}\right) \int_{\lambda}^{\mu} \frac{d \tau}{\tau} \frac{\alpha_{s}(\tau)}{\pi}\right] \phi_{1}(\mu / \lambda, \theta) .
$$


According, now, to the guidelines set by our discussion in subsection 3.1 we proceed to determine that

$$
\frac{d}{d \ln Q^{2}} \ln \left(W_{1}^{(i)}\right)_{S O F T}=-2 c_{F} \ln \left(\frac{2 Q^{2}}{m^{2}}\right) \int_{\lambda}^{\mu} \frac{d \tau}{\tau} \frac{\alpha_{s}(\tau)}{\pi}+\frac{1}{\ln Q^{2}}
$$

whereupon, with the aid of (47), we deduce

$$
\frac{d}{d \ln \mu} \frac{d}{d \ln Q^{2}} \ln \left(W_{1}^{(i)}\right)_{H A R D}=\frac{2 c_{F}}{\pi} \alpha_{s}(\mu)
$$

or

$$
\frac{d}{d \ln Q^{2}} \ln \left(W_{1}^{(i)}\right)_{H A R D}=-2 c_{F} \int_{\mu}^{|Q|} \frac{d \tau}{\tau} \frac{\alpha_{s}(\tau)}{\pi}+\mathcal{R}\left(\alpha_{s}(Q)\right)
$$

The above result when put together with (73) gives

$$
\frac{d}{d \ln Q^{2}} \ln \left(W_{1}^{(i)}\right)=-2 c_{F} \int_{\lambda^{2}}^{\mu^{2}} \frac{d \tau}{2 \tau} \frac{\alpha_{s}(\tau)}{\pi}-2 c_{F} \int_{\mu^{2}}^{Q^{2}} \frac{d \tau}{2 \tau} \frac{\alpha_{s}(\tau)}{\pi}+\mathcal{R}\left(\alpha_{s}(Q)\right),
$$

which leads to

$$
W_{1}^{(i)}=\mathcal{L}\left[\alpha_{s}\left(Q^{2}\right)\right] \exp \left[-2 c_{F} \int_{\mu^{2}}^{Q^{2}} \frac{d \tau}{2 \tau} \frac{\alpha_{s}(\tau)}{\pi} \ln \frac{Q^{2}}{\tau}\right]
$$

But

$$
\begin{aligned}
2 c_{F} \int_{\mu^{2}}^{Q^{2}} \frac{d \tau}{2 \tau} \frac{\alpha_{s}(\tau)}{\pi} \ln \frac{Q^{2}}{\tau}= & \frac{4 c_{F}}{\beta_{o}}\left[\ln \left(\frac{Q^{2}}{\Lambda^{2}}\right)-\ln \ln \left(\frac{Q^{2}}{\Lambda^{2}}\right)\right. \\
& \left.\ln \left(\frac{Q^{2}}{\Lambda^{2}}\right) \ln \ln \left(\frac{\lambda^{2}}{\Lambda^{2}}\right)-\ln \left(\frac{Q^{2}}{\Lambda^{2}}\right)\right],
\end{aligned}
$$

which explicitly exhibits Sudakov behaviour for the $W_{1}^{(i)}$ through its leading term [36].

Some comments are in order at this point. Beginning with technical issues, let us first notice that, in the asymptotic regime under consideration, $W_{1}^{(a)} \sim W_{1}^{(b)}$. This means that the Sudakov behaviour of the $W_{1}^{(i)}$ passes on to the full expression for $\left(W_{1}\right)_{j j^{\prime}}^{i i^{\prime}}$. Moreover, nothing changes if one goes to the corresponding quantity $\left(E_{1}\right)_{j j^{\prime}}^{i i^{\prime}}$ associated with the connected fourpoint function since the two disjoint cusped line configurations, which make the difference between the connected and disconnected expressions, will each provide similarly suppressing Sudakov form factors. Finally, particle exchange in the final states, which cannot be $a$ priori excluded from consideration in the fixed angle case, simply permutes the $s$ with the $t$ variable without affecting our results. Let us also note, on the technical front, that our 
final expressions contain non-leading terms whose assesment should provide interesting new information.

From a physical standpoint the message to be drawn from the Sudakov behaviour that has been extracted for the fixed angle amplitude amounts to the standard realization that the larger the momentum transfer between the colliding particles the smaller the probability for the process to remain exclusive.

\section{Concluding Remarks}

The worldline casting of gauge theories with spin-1/2 matter fields has as its basic feature the space-time setting within which physical quantities are described. Both particle propagation and dynamics, the latter in the form of Wilson lines, are embodied in space-time paths. Generically, of course, all possible contours enter the path integral. By restricting ourselves to paths that are straight almost everywhere, we were able to achieve a sharp factorization of a soft, relevant to the process, sector at the fundamental field theoretical level.

For the case where no cusps, to break the straight contours, are present we are dealing with situations where the no impulse approximation holds throughout, equivalently the 'soft' subsector represents the full field theory. Nevertheless, it is possible to determine anomalous dimensions governing processes in this domain, an occurence which reflects the fact that from the viewpoint of the IR cutoff $\lambda$ the 'upper roof' $\Lambda$ of the soft subsector appears to be infinite. For the four-point, forward scattering process considered in this paper the corresponding RG considerations led to gluon reggeization.

For processes in which cusped configurations make their entrance a non-negligible momentum transfer takes place on the basis of a sudden impulse approximation. This time the RG running aquires the standard interpretation of a factorization between soft and hard physics, within the isolated, with respect to the considered energy range, subtheory. This situation is analogous to the operator product expansion that separates Wilson coefficients (hard factors) from operator expectation values (soft factors). In the fixed angle scattering regime that we considered in section 3, the end result was the emergence of Sudakov behavior for the amplitude.

We hope to have sufficiently illustrated the efficiency by which the factorization of soft 
physics can be attained within the worldline casting of non-abelian gauge theories. Morever, once familiarization with computational methodology and procedure is aquired, one realizes that the worldline handling of soft subsectors involves more or less similar mathematical expressions, irrespective of the process one studies. Thus, along with the conceptual simplicity regarding the factorization strategy there are additional advantages, of practical nature, to the proposed approach as well.

Clearly, the results we have exhibited are valid to $\mathcal{O}(\Lambda)$. In the OPE language this amounts to leading twist. Non leading contributions are lurking in our expressions and we intend to study their implications in future work. More interesting is the question concerning the relation between the factorization advocated in this paper and the standard factorization widely discussed in the literature with basic reliance on Feynman diagrammatic logic, especially in connection with exclusive processes. We intend to report on this issue in a forthcoming paper. 


\section{Appendix}

We trace the steps which take us from (66) to (69) and (70).

We define

$$
\hat{C}(\tilde{A}) \equiv \exp \left[-\tilde{A} \int_{\lambda}^{\mu} \frac{d \tau}{\tau} \frac{\alpha_{s}(\tau)}{\pi}\right],
$$

where $\tilde{A}$ stands for the $2 \times 2$ matrix given by (64).

The following identity holds

$$
\begin{aligned}
\hat{C}(\tilde{A}) & =\frac{A_{+}-\tilde{A}}{A_{+}-A_{-}} \hat{C}\left(A_{-}\right)-\frac{A_{-}-\tilde{A}}{A_{+}-A_{-}} \hat{C}\left(A_{+}\right) \\
& =\frac{1}{A_{+}-A_{-}}\left[A_{+} \hat{C}\left(A_{-}\right)-A_{-} \hat{C}\left(A_{+}\right)\right]+\tilde{A} \frac{1}{A_{+}-A_{-}}\left[\hat{C}\left(A_{+}\right)-\hat{C}\left(A_{-}\right)\right],
\end{aligned}
$$

where $A_{+}$are the eigenvalues of $\tilde{A}$.

We write

$$
\hat{C}(\tilde{A})_{11}=X+Y A_{11}, \quad \hat{C}(\tilde{A})_{12}=Y A_{12},
$$

where

$$
X \equiv \frac{1}{A_{+}-A_{-}}\left[A_{+} \hat{C}\left(A_{-}\right)-A_{-} \hat{C}\left(A_{+}\right)\right], \quad Y \equiv \frac{1}{A_{+}-A_{-}}\left[\hat{C}\left(A_{+}\right)-\hat{C}\left(A_{-}\right)\right] .
$$

It follows from Eq. (66) in the text, that

$$
\left(W_{1}^{(a)}\right)_{S O F T}=N^{2} X+Y N\left(N A_{11}+A_{12}\right)
$$

and

$$
\left(W_{1}^{(b)}\right)_{S O F T}=N X+Y N\left(A_{11}+N A_{12}\right)
$$

Referring to Eqs. (54), (55), (60), (61) in the text and taking into account the asymptotic conditions $(67,(68)$ we determine

$$
\begin{aligned}
& A_{11} \simeq 2 c_{F} \ln \left(\frac{2 Q^{2}}{m^{2}}\right)+2 c_{F} \ln \left(\sin ^{2} \frac{\theta}{2}\right)-\frac{1}{N} \ln \left(\cos ^{2} \frac{\theta}{2}\right)-\frac{i \pi}{N} \\
& A_{12} \simeq i \pi+\ln \left(\cos ^{2} \frac{\theta}{2}\right) \\
& A_{21} \simeq i \pi+\ln \left(\sin ^{2} \frac{\theta}{2}\right) \\
& A_{22} \simeq 2 c_{F} \ln \left(\frac{2 Q^{2}}{m^{2}}\right)+2 c_{F} \ln \left(\cos ^{2} \frac{\theta}{2}\right)-\frac{1}{N} \ln \left(\sin ^{2} \frac{\theta}{2}\right)-\frac{i \pi}{N} .
\end{aligned}
$$


We therby obtain

$$
\begin{aligned}
A_{+} & \simeq 2 c_{F} \ln \left(\frac{2 Q^{2}}{m^{2}}\right)+\left(c_{F}-\frac{1}{2 N}\right) \ln \left(\frac{1}{4} \sin \theta\right)-\frac{i \pi}{N} \\
& +\frac{1}{2} \sqrt{N^{2} \ln ^{2}\left(\frac{1}{4} \sin \theta\right)-4 \pi^{2}+4 i \pi \sin \left(\frac{1}{4} \sin \theta\right)}
\end{aligned}
$$

Substituting into the expressions for $X$ and $Y$ we find

$$
X=\exp \left[-2 c_{F} \ln \left(\frac{2 Q^{2}}{m^{2}}\right) \int_{\lambda}^{\mu} \frac{d \tau}{\tau} \frac{\alpha_{s}(\tau)}{\pi}\right]\left\{2 c_{F} \ln \left(\frac{2 Q^{2}}{m^{2}}\right) \phi_{1}(\mu / \lambda, \theta)+\phi_{2}(\mu / \lambda, \theta)\right\}
$$

and

$$
Y=\exp \left[-2 c_{F} \ln \left(\frac{2 Q^{2}}{m^{2}}\right) \int_{\lambda}^{\mu} \frac{d \tau}{\tau} \frac{\alpha_{s}(\tau)}{\pi}\right] \phi_{1}(\mu / \lambda, \theta)
$$

where

$$
\phi_{1}(\mu / \lambda, \theta) \equiv \frac{1}{C_{+}-C_{-}}\left[e^{-C_{-} \int_{\lambda}^{\mu} \frac{d \tau}{\tau} \frac{\alpha_{s}(\tau)}{\pi}}-e^{-C_{+} \int_{\lambda}^{\mu} \frac{d \tau}{\tau} \frac{\alpha_{s}(\tau)}{\pi}}\right]
$$

and

$$
\phi_{2}(\mu / \lambda, \theta) \equiv \frac{1}{C_{+}-C_{-}}\left[C_{+} e^{-C_{-} \int_{\lambda}^{\mu} \frac{d \tau}{\tau} \frac{\alpha_{s}(\tau)}{\pi}}-C_{-} e^{-C_{+} \int_{\lambda}^{\mu} \frac{d \tau}{\tau} \frac{\alpha_{s}(\tau)}{\pi}}\right]
$$

Substituting into the relations giving the $\left(W_{1}^{(i)}\right)_{S O F T}$ and keeping only the $Q^{2}$-dependent part of the resulting expressions we finally arrive at Eqs. (69) and (70) given in the text. 


\section{References}

[1] H. Cheng and T. T. Wu, Expanding Protons: Scattering at High Energies (M. I. T. Press, Cambridge, MA, 1987).

[2] A. I. Karanikas and C. N. Ktorides, Phys. Lett B 275, 403 (1992); Int. J. Mod. Phys. A7, 5563 (1992).

[3] A. I. Karanikas and C. N. Ktorides, Phys. Rev. D 52, 5883 (1995).

[4] G. C. Gellas, A. I. Karanikas and C. N. Ktorides, Ann. Phys. (NY), 228 (1997).

[5] G. C. Gellas, A. I. Karanikas, C. N. Ktorides and N. G. Stefanis, Phys. Lett. B 412, 95 (1997).

[6] G. C. Gellas, A. I. Karanikas and C. N. Ktorides, Phys. Rev. D 57, 3763 (1998).

[7] A. V. Radyushkin, Acta Phys. Polon. B 15, 403 (1984).

[8] N. Isgur and C. H. Llewellyn Smith, Phys. Rev Lett. 52, 1080 (1984); Nucl. Phys. B 317, 526 (1989).

[9] J. Botts and G. Sterman, Nucl. Phys. B 325, 62 (1989)

[10] P. Kroll and R. Jacob, Phys. Lett. B 315, 463 (1993); B 319. 545(E) (1993).

[11] V. L. Chernyak nd A. R. Zhitnitsky, Nucl. Phys. B 246, 52 (1984): Phys. Rep. 112, $173(1984)$.

[12] M. Gari and N G. Stefanis, Phys. Lett. B 175, 462 (1986).

[13] V. N. Gribov and L. N. Lipatov, Sov. J. Nucl. Phys. 15, 438 (1972); 15, 675 (1972); Y. I. Dokshitzer, Sov. Phys. JETP 46, 641 (1977); G. Altarelli and G. Parisi, Nucl. Phys. B 129, 298 (1977).

[14] E. A. Kuraev, L. N. Lipatov and V. S. Fadin, Sov. Phys. JETP 44, 443 (1976); 45, 199 (1977); Y. Y. Balitski and L. N. Lipatov, Sov. J. Nucl. Phys. 28, 822 (1978). 
[15] E. S. Fradkin, Nucl. Phys. B 76, 588 (1966).

[16] H. M. Fried, Basics of Functional Methods and Eikonal Models (Editions Frontieres, Gif-Sur Yvette, France, 1990).

[17] M. J. Strassler, Nucl. Phys. B 385, 145 (1992).

[18] M. G. Schmidt and C. Schubert Phys. Lett. B 331, 68 (1994); M. Reuter, M. G. Schmidt and C. Schubert, Ann. Phys. (N.Y.), 259, 313 (1997).

[19] A. M. Polyakov, Nucl. Phys. B 164, 171 (1980).

[20] R. A. Brandt, F. Neri and M-A. Sato, Phys. Rev. D 24, 879 (1981).

[21] R. A. Brandt, A. Gocksch, F. Neri and M-A. Sato, Phys. Rev. D 26, 3611 (1982).

[22] V. S. Dotsenko and S. N. Vergeles, Nucl. Phys. B 169, 204 (1981).

[23] N. S. Craigie and H. Dorn, Nucl. Phys. B 185, 204 (1981).

[24] J. C. Collins and D. E. Soper, Nucl. Phys. B 194, 445 (1982).

[25] G. P. Korchemsky and A. V. Radyushkin, Phys. Lett. B 279, 359 (1992).

[26] G. P. Korchemsky, Phys. Lett. B 325, 459 (1994); I. A. Korchemskaya and G. P. Korchemsky, Nucl. Phys. B 437, 127 (1994).

[27] V. Sudakov, Sov. Phys. JETP 3, 65 (1956).

[28] R. Jackiw, Ann. Phys. (N.Y.) 48, 292 (1968).

[29] L. N. Lipatov, Nucl. Phys. B 309, 379 (1988).

[30] L. N. Lipatov, Proceedings of the IV ITEP Winter School, (1982).

[31] L. N. Lipatov, Nucl. Phys. B 365, 614 (1991).

[32] H. Verlinde and E. Verlinde, preprint hep-th/9302104 (1993).

[33] L. N. Lipatov, Nucl. Phys. B 452, 369 (1995). 
[34] I. Balitsky, Nucl. Phys. B 463, 99 (1996).

[35] F. Botterweck te al., Z. Phys. C 51, 541 (1991); S. Banerjee et al., Phys. Lett. B 305, 182 (1993); A. Belogianni et al., Phys. Lett. B 408, 487 (1997).

[36] A. Sen, Phys. Rev. D 28860 (1983).

[37] H. Bloch and A. Nordsieck, Phys. Rev. 52, 54 (1937).

[38] A. Kernemann and N. G. Stefanis, Phys. Rev. D 40, 2103 (1989). 


\section{FIGURE CAPTIONS}

Fig. 1a: A Cheng-Wu tower entering the (forward) fermion-fermion scattering process in QED. The unitarity cut (dashed line) reveals a fragmented profile of an electron (positron).

Fig. 1b: Depiction of a forward, high-energy fermion-fermion scattering process in a non-abelian gauge field theory. Gluons linking the scattered particles reggeize while the fragmented profile of the latter is presented by gluons exchanged between reggeons.

Fig. 2a: Worldline depiction of forward elastic scattering between spin-1/2 matter particles. Closest distance of approach (impact parameter) is $z$.

Fig. 2b: Double cusped configuration which mixes with the four-point function associated with the forward scattering process, depicted in Fig. 2a, under renormalization group running.

Fig. 3a: Wilson loop version of Fig. 2a, corresponding to an on mass shell situation.

Fig. 3b: Wilson loop version of Fig. 2b, corresponding to an on mass shell situation.

Fig. 4a: Worldline depiction of a (near) forward process which excludes photon exchange within a region of size $T$ around the point of closest approach.

Fig. 4b: Feynman diagrammatic representation of the situation depicted in Fig. 4a.

Fig. 5a: Worldline depiction of fermion-fermion scattering at fixed angle, in the sudden implulse approximation.

Fig. 5b: Worldline depiction of the, double-cusped, contour associated with the operator that mixes with the fixed angle scattering one, under renormalization group running. 


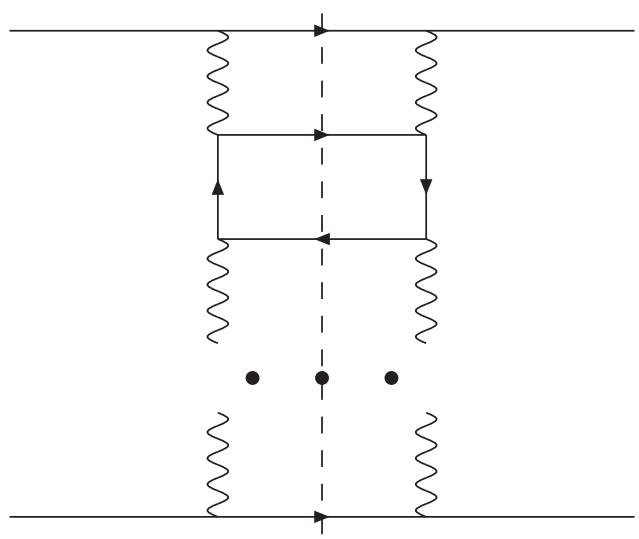

Fig 1a

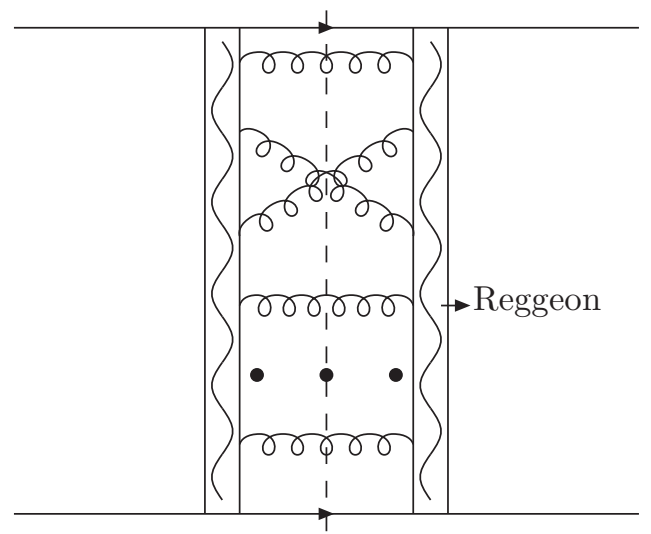

Fig. 1b

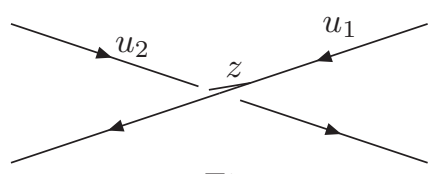

Fig. $2 \mathrm{a}$

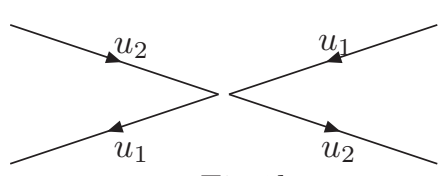

Fig.2b

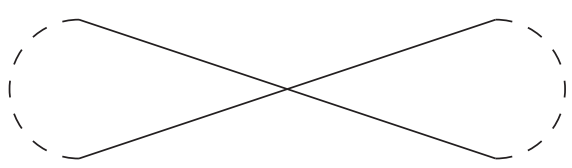

Fig. 3a

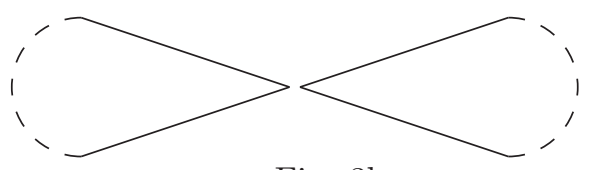

Fig. 3b 


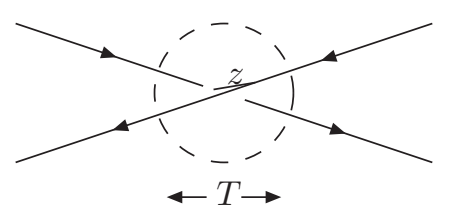

Fig. 4a

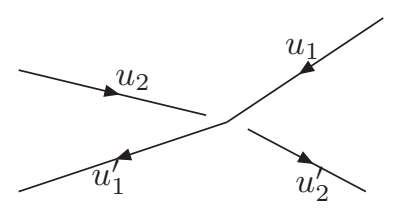

Fig. 5a

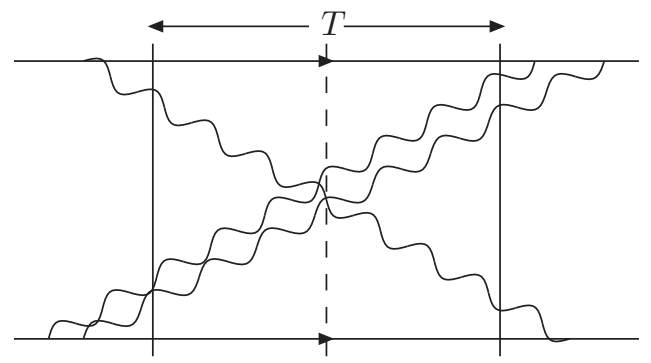

Fig. 4b

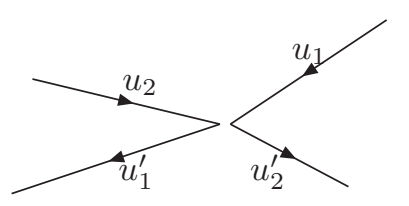

Fig. 5b 